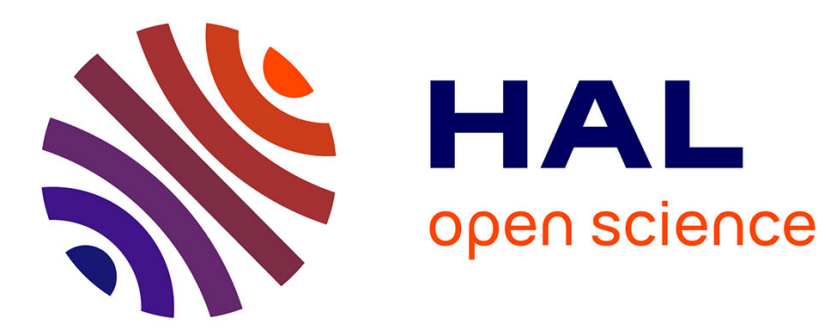

\title{
The electrical conductivity of TTF-TCNQ under pressure
}

\author{
J.R. Cooper, D. Jérome, M. Weger, S. Etemad
}

\section{To cite this version:}

J.R. Cooper, D. Jérome, M. Weger, S. Etemad. The electrical conductivity of TTF-TCNQ under pressure. Journal de Physique Lettres, 1975, 36 (9), pp.219-223. 10.1051/jphyslet:01975003609021900 . jpa-00231192

\section{HAL Id: jpa-00231192 https://hal.science/jpa-00231192}

Submitted on 1 Jan 1975

HAL is a multi-disciplinary open access archive for the deposit and dissemination of scientific research documents, whether they are published or not. The documents may come from teaching and research institutions in France or abroad, or from public or private research centers.
L'archive ouverte pluridisciplinaire HAL, est destinée au dépôt et à la diffusion de documents scientifiques de niveau recherche, publiés ou non, émanant des établissements d'enseignement et de recherche français ou étrangers, des laboratoires publics ou privés. 


\title{
THE ELECTRICAL CONDUCTIVITY OF TTF-TCNQ UNDER PRESSURE
}

\author{
J. R. COOPER $(*)$, D. JÉROME and M. WEGER $(* *)$ \\ Laboratoire de Physique des Solides $\left({ }^{* *}\right)$, Université Paris-Sud, Centre d'Orsay, 91405 Orsay, France \\ and
}

S. ETEMAD

I. B. M. Thomas J. Watson Research Center, Yorktown Heights, N. Y. 10598 U. S. A.

(Reçu le 2 mai 1975, accepté le 2 juin 1975)

\begin{abstract}
Résumé. - Nous présentons des mesures de conductivité électrique suivant l'axe $b$ dans des monocristaux de TTF-TCNQ entre 20 et $300 \mathrm{~K}$ sous pression hydrostatique jusqu'à 6 kbar. Deux anomalies de conductivité existent. La température de la transition métal-isolant de $54 \mathrm{~K}$ à pression nulle augmente légèrement sous pression (en accord avec les résultats de Chu $e t$ al.). Nous trouvons que la température de l'anomalie de $38 \mathrm{~K}$ décroît faiblement sous pression. Compte tenu de l'augmentation appréciable de la largeur de la bande de conduction sous pression, nous ne pouvons interpréter les résultats observés au moyen des théories habituelles de la transition de Peierls.

Abstract. - We report $b$-axis electrical conductivity data for TTF-TCNQ single crystals from 20-300 K under hydrostatic pressures of up to $6 \mathrm{kbar}$. There are two anomalies in the conductivity. Under pressure, the temperature of the well-known metal-insulator transition at $54 \mathrm{~K}$ increases slightly (in agreement with the earlier work of Chu et al.). We find that the temperature of the lower anomaly at $38 \mathrm{~K}$ decreases slowly with pressure. Since there is evidence that the conduction electron bandwidths increase substantially in the above pressure range it is not easy to interpret these results in terms of current theories of the Peierls transition.
\end{abstract}

1. Introduction. - We have measured the $b$-axis conductivity of three single crystals of the organic charge transfer salt TTF-TCNQ from 20-300 K under hydrostatic (He gas) pressures of up to $6 \mathrm{kbar}$. The main reason for this study was to clarify some results reported a year ago [1] which seemed to indicate that there were $t w o$ transitions below $60 \mathrm{~K}$ in this compound and what is more important, that one of them was strongly pressure dependent. Although we have mainly investigated the region below $60 \mathrm{~K}$ we will also briefly mention the effect of pressure at higher temperatures in the metallic region.

2. Experimental. - The samples were prepared at the I. B. M. Laboratory. Results for the D. C. conductivity at ambient pressure for samples prepared in the same way with similar electrical contacts have just been reported in two publications by Etemad et al. [2, 3]. The results reported here were obtained using a

$\left(^{*}\right)$ On leave from : Institute of Physics of the University, Zagreb, Yugoslavia.

$(* *)$ Permanent address : The Hebrew University of Jerusalem, Racah Institute of Physics, Israël.

$(* * *)$ Laboratoire associé au C. N. R. S.
4 contact A. C. method at a frequency of $70 \mathrm{~Hz}$ and measuring currents from 1 to $5 \mu \mathrm{A}$ R. M. S. A copperconstantan thermocouple mounted inside the pressure bomb was used to determine the sample temperature. On most runs its calibration was checked against an external platinum thermometer. Resistance-temperature curves were continuously displayed on an $\mathrm{X}-\mathrm{Y}$ recorder while slowly cooling $(\sim 2 \mathrm{~K} / \mathrm{min})$ or warming $(\sim 0.5 \mathrm{~K} / \mathrm{min})$ the large pressure bomb containing the sample. This method is well suited for the detection of any anomalies in the $R(T)$ curve but there are rather large uncertainties in the sample temperature (at least $\pm 1 \mathrm{~K}$ ) and the resistance values are only accurate to a few percent.

3. Description of experimental results. - The main feature that we notice in the $R$ vs. $T$ curves, examples of which are shown in figure 1 , is that they possess anomalies at two temperatures, $T_{\mathrm{HIGH}}=54 \pm 1 \mathrm{~K}$ and $T_{\text {Low }}=38 \pm 1 \mathrm{~K}$, in agreement with the results of reference [1] and more recent work at ambient pressure [3]. Anomalies at these temperatures were observed in all samples investigated, and as shown in figure 2 these temperatures depend only slightly on the pressure, namely $\mathrm{d} T_{\mathrm{HIGH}} / \mathrm{d} P=0.7 \pm 0.3 \mathrm{~K} / \mathrm{kbar}$, 


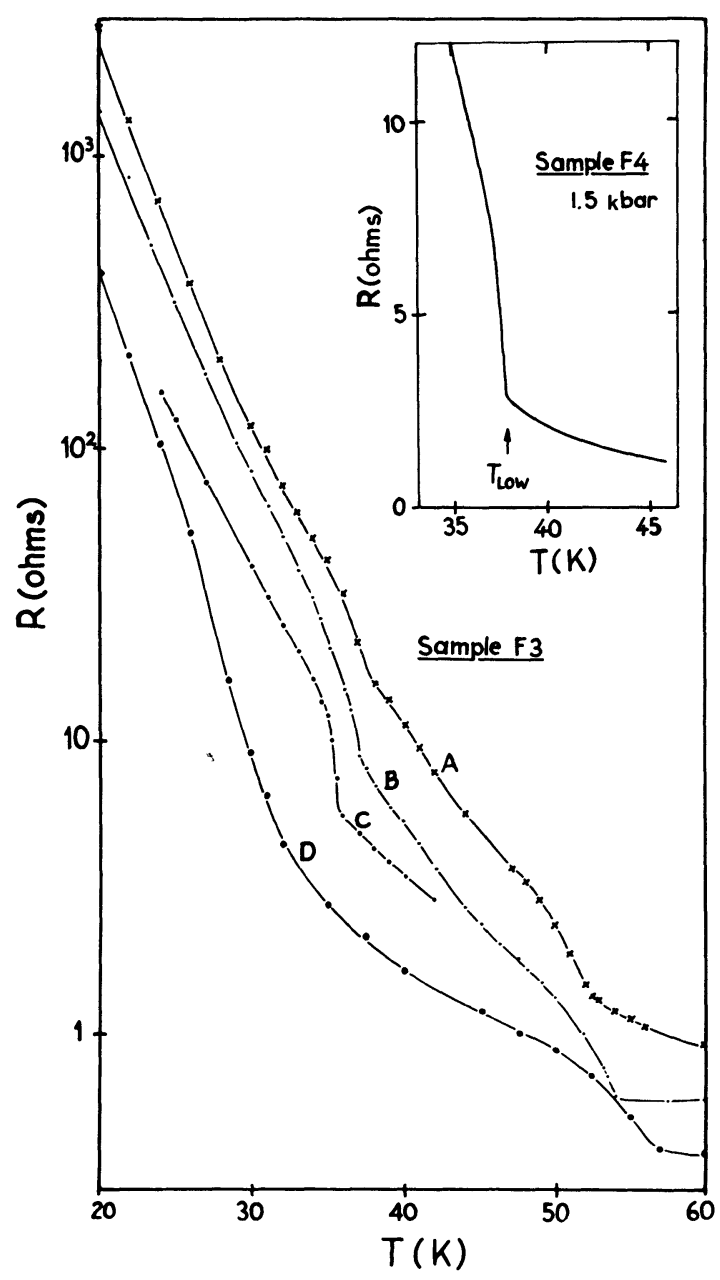

FIG. 1. - Resistance-temperature curves for a TTF-TCNQ sample at several pressures. A 100 bar, B ambient pressure, C 2 kbar, D 5 kbar. Order of measurement was BDCA. The curves shown were recorded as $T$ increases. Inset : Tracing of a recorded $R(T)$ curve showing the anomaly at $T_{\text {LOW }}$ in more detail.

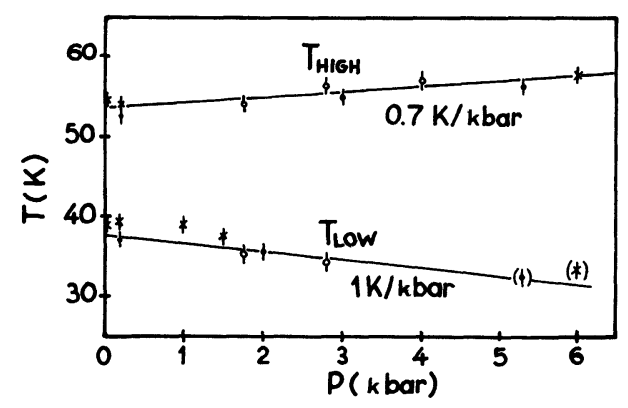

Fig. 2. - Transition temperatures $T_{\text {HiGH }}$ and $T_{\text {LOw }}$ versus pressure for samples F 20, F 30 and F $4 X$. Small differences between samples are ascribed to systematic errors in the temperature scale. The transitions at $T_{\text {Low }}$ were not sharp at pressures of 5 and $6 \mathrm{kbar}$.

$\mathrm{d} T_{\text {Low }} / \mathrm{d} P=-1.0 \pm 0.4 \mathrm{~K} / \mathrm{kbar}$. This pressure dependence of $T_{\text {HIGH }}$ is in agreement with the results of $\mathrm{Chu}$ et al. [4]. (The transition at $T_{\text {Low }}$ was not reported by them.) The small pressure dependence of $T_{\text {Low }}$ is in disagreement with the interpretation suggested in reference [1].

The anomaly at $T_{\text {HIGH }}$ manifests itself as a discontinuous change in the slope of the $R$ vs. $T$ curve, from a nearly temperature independent resistance just above $T_{\text {HIGH }}$ (see in particular sample F 3 , curve B), to a slope of order

$$
\frac{1}{R} \frac{\mathrm{d} R}{\mathrm{~d} T}=-0.25 \pm 0.1(\mathrm{~K})^{-1}
$$

just below $T_{\text {HIGH }}$. The larger slopes, -0.3 to $-0.35(\mathrm{~K})^{-1}$ are observed at low pressure, and the smaller slopes, -0.15 to $-0.2(\mathrm{~K})^{-1}$ are observed at pressures of order 5-6 kbar. Below about $50 \mathrm{~K}$ the slope again decreases slightly.

The anomaly at $T_{\text {LOw }}$ manifests itself as an S-shaped curve, or even a discontinuity, in the $R$ vs. $T$ curve. The discontinuity is particularly sharp at pressures of 1.5 (sample F 4, inset to Fig. 1) to 2 (sample F 3, curve C) kbar. (See also ref. [1]). At low pressure (0-1 kbar) the anomaly is smaller, and at higher pressures (5-6 kbar) it is again smeared out ; however, at these pressures the helium is solid so we cannot be certain that the pressure is absolutely hydrostatic and uniform. (These results are also in agreement with those of ref. [1].) In the pressure range $1.5-2 \mathrm{kbar}$, the discontinuity in the resistance is roughly an increase by a factor of 3 , as the temperature is lowered.

Temperature and pressure cycling has some effect on the curves, after several (6-10) cycles, the transitions are somewhat smeared out, and the measured resistivity increases. However the values of $T_{\mathrm{HIGH}}$ and $T_{\mathrm{LOW}}$ are not affected by the thermal cycling.

Above $T_{\text {HIGH }}$ all samples are metallic i. e. $R$ increases with $T$. We have tried two ways of fitting the measured $T$ dependence under pressure. If the relationship :

$$
R=R_{0}+R_{1}\left(\frac{T}{300}\right)^{\lambda}
$$

proposed by Groff et al. [5] is used then at ambient pressure the values of $\lambda$ for the three samples lie between 2.2 and 2.5 and fall slightly to $1.8-2.0$ at $6 \mathrm{kbar}$. The parameters $R_{0}$ and $R_{1}$ are more strongly pressure dependent, typically $R_{0}$ falls by a factor of 2 and $R_{1}$ by a factor of 3 from 0-6 kbar.

However, the physical significance of the above formula is not clear and as noted in reference 2 a simple $T^{2}$ fit :

$$
R=A+B T^{2}
$$

is probably preferable.

Reasonably good $T^{2}$ fits were obtained for all samples in the pressure range studied, some examples are given in figure 3.

At ambient pressure there is certainly an upward deviation from a $T^{2}$ law for all three samples, consistent with the fact that $\lambda \simeq 2.3$ is really a better fit. 


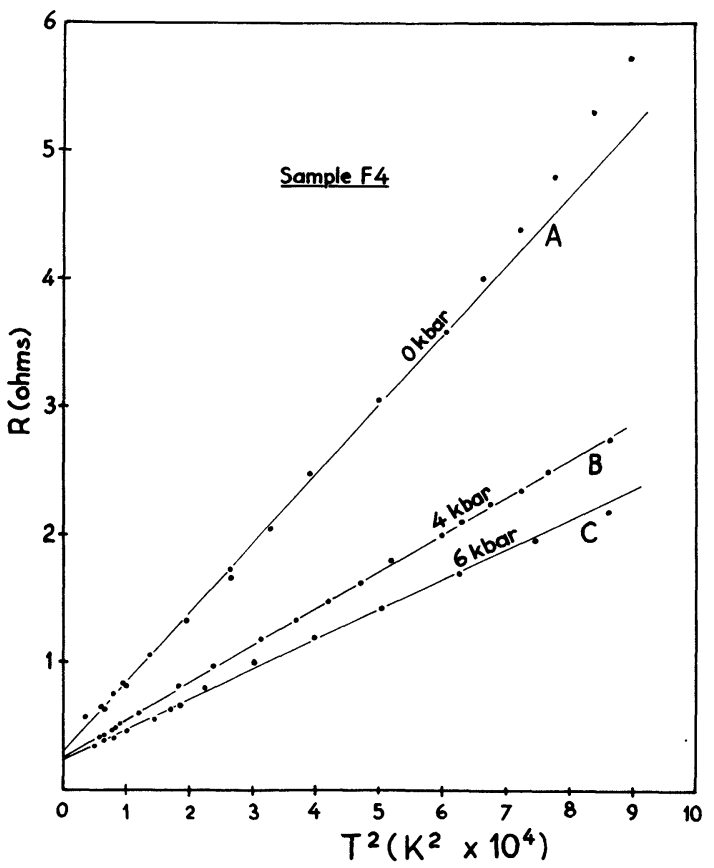

Fig. 3. - Examples of $T^{2}$ fits in the region $70-300 \mathrm{~K}$.

One possible reason for this deviation may be the thermal expansion of the lattice. X-ray studies [17] indicate that the spacing of the TCNQ molecules along the $b$-axis increases by $2 \%$ from $100-300 \mathrm{~K}$. According to the estimate of the compressibility given below and the measured pressure dependence of the resistance such a change (equivalent to a decrease in pressure of $\sim 0.6 \mathrm{kbar}$ ) could give $15 \%$ deviations from a $T^{2}$ law. Another possible cause of deviations from a $T^{2}$ law could be imperfect contacts, resulting in a small temperature dependence of the effective cross-sectional area of the sample as the anisotropy varies with temperature. This effect would presumably be reduced by application of pressure, in agreement with experimental observations.

We may note that at room temperature the effect of pressure is reproducible from sample to sample. For the three samples studies here and for those of references [1] and [4] the conductivity increases with pressure (approximately linearly) by a factor of 2 from ambient to 3-4 kbar. The coefficient $A$ in eq. (2) (i. e. the residual resistance) is less strongly pressure dependent but with our experimental technique where pressure is applied at room temperature and released at low temperature, it is not possible to properly separate the effect of pressure and the effect of thermal cycling on the coefficient $A$.

For all the measurements made in the temperature interval $T_{\mathrm{HIGH}}<T<300 \mathrm{~K}$ the impedance was resistive (at the frequency of $70 \mathrm{~Hz}$ employed here) and the un-nested voltage small (of order $5 \%$ or less than the nested voltage).

For $20<T<T_{\text {Low }}$ the resistance can be fitted to the relationship $R=R^{*} \exp (\Delta / T)$ with $\Delta=210 \pm 10 \mathrm{~K}$ for samples F 3 and F 4 in reasonable agreement with the more precise results of Etemad et al. [3] at ambient pressure in this temperature interval. However, this value of $\Delta$ is much higher than the activation energy found below $8 \mathrm{~K}$ in some earlier work [6], so the latter may not necessarily represent the true activation energy for the conductivity. This point could be checked by further work at ambient pressure. $\Delta$ decreases slightly with pressure, $\mathrm{d} \Delta / \mathrm{d} P=-10 \pm 5 \mathrm{~K} / \mathrm{kbar}$. $R^{*}$ is pressure independent to within the experimental accuracy. The impedance is resistive, and the un-nested voltage is small (of order $10 \%$ or less of the nested voltage in all samples).

In the region $T_{\text {LOw }}<T<T_{\text {HIGH }}$, the behavior of the resistivity is more complicated. The $R$ vs. $T$ curve is somewhat S-shaped. $|\mathrm{d} R / \mathrm{d} T|$ is large for

$$
50<T<T_{\text {HIGH }},
$$

and small for $T \approx 40-45 \mathrm{~K}$. The curvature is somewhat different in different samples (for example, in sample $\mathrm{F} 2$, and the sample of ref. [1], $\mathrm{d} R / \mathrm{d} T$ changes sign around $40 \mathrm{~K}$ ). The curvature changes somewhat with pressure (i. e. for $T=40 \mathrm{~K}|\mathrm{~d} R / \mathrm{d} T|$ ) becomes smaller at pressures of 1.5-6 kbar, and (in some samples at least), there is some effect of thermal cycling on the slope. In sample F 2, where these effects are particularly large, also a large un-nested voltage was observed in this region, and the impedance was not resistive (at $70 \mathrm{~Hz}$ ).

4. Estimate of the compressibility of TTF-TCNQ. For a quantitative discussion of the effect of pressure, it is necessary to know the elastic constants of TTFTCNQ, especially the relative change with pressure of the molecular spacing along the conducting direction, namely the logarithmic derivative $\alpha \equiv \mathrm{d} \cdot \ln b / \mathrm{d} P$. The compressibilities of ordinary organic crystals such as anthracene or napthalene are such that 1 kbar produces a volume change of $1-1.5 \%$ [18] which leads to $\alpha \simeq 0.3$ to $0.5 \%$ per kbar. However measurements of Youngs modulus in TTF-TCNQ [16] show that it is even softer than these materials. By neglecting the offdiagonal elastic constants an upper limit of 2 to $3 \%$ per kbar can be estimated for $\alpha$ from the results of reference [16]. Since in a tight-binding approximation the intermolecular transfer integrals depend exponentially on $b$ and the value of the exponent is $\simeq-4$ for the TCNQ chain [14] then it is probable that the TCNQ bandwidth increases by $8-12 \%$ per kbar in TTF-TCNQ. This is a factor of 2 or 3 smaller than the measured increase in the conductivity at room temperature. The TTF bandwidth is probably somewhat smaller [12] so it should increase slightly faster under pressure, approximately $15 \% / \mathrm{kbar}$.

5. Discussion. - 5.1. The most striking result of the present work seems to be the unambiguous observation of a second transition in TTF-TCNQ, at $38 \mathrm{~K}$ (confirming the preliminary report of ref. [1] and 
those of ref. [3]), in addition to the well known transition at $54 \mathrm{~K}$ [7]. An anomaly in the anisotropy $\sigma_{\|} / \sigma_{\perp}$ in the vicinity of $T_{\text {LOW }}$ has been reported previously [8]. This anomaly has the shape of a sharp peak in $\sigma_{\|} / \sigma_{\perp}$. If the electrical contacts are not ideally located, the measured resistance $R$ is affected by the anisotropy [9]. However, a peak in $\sigma_{\|} / \sigma_{\perp}$ cannot give rise to a spurious step in $R$, since $R=\left(\sigma_{\|}\right)^{-1} f\left(\sigma_{\|} / \sigma_{\perp}\right)$, and since $f$ is a single-valued function, a peak in $\sigma_{\|} / \sigma_{\perp}$ can give rise only to a peak (or minimum) in $R$, but not to a step discontinuity. Furthermore it is very unlikely that smooth activated temperature dependences of $\sigma_{\|}$and $\sigma_{\perp}$ could give rise to such a sharp increase in $R$. However the transition at $T_{\text {Low }}$ reported here may be the cause of the anomaly in the anisotropy observed in the same temperature region.

5.2 This work establishes (together with ref. [4]) that the temperatures of the transitions $T_{\mathrm{HIGH}}$ and $T_{\text {LOw }}$ are only weakly pressure dependent. This observation has considerable theoretical significance. Several theories attribute the metal-insulator transition in TTFTCNQ to a Peierls transition at $T_{\mathrm{p}}$. Either $T_{\mathrm{HIGH}} \approx T_{\mathrm{p}}$ [10], or, $T_{\mathrm{HIGH}} \approx \frac{1}{4} T_{\mathrm{p}}$ due to interchain correlations [11]; alternatively, the transition is associated with a gap in the band structure [12]. For any of these models, $T_{\text {HIGH }}$ should be strongly pressure dependent, since the Peierls transition temperature is given by [13] $T_{\mathrm{p}} \approx E_{\mathrm{F}} \exp (-1 / \lambda)$ and $E_{\mathrm{F}}$ (and probably $\lambda$ too) must be pressure dependent to account for the strong pressure dependence of the resistivity in the metallic state [1]. The interchain transfer integrals [12], [14] must also be strongly pressure dependent due to the change in overlap with volume, even more so than the intrachain ones estimated in the previous section (provided that the compressibility perpendicular to the chains is also large).

Moreover, recent X-ray work $\left({ }^{1}\right)$ indicates that there are incommensurate one-dimensional fluctuations in the region above $40 \mathrm{~K}$ and that the transition at $T_{\text {Low }}$ may correspond to the appearance of the associated superstructure, i. e., three-dimensional ordering. So, undoubtedly there will be renewed interest in the transition at $T_{\text {LOw }}$. The observation that both $T_{\text {HIGH }}$ and $T_{\text {Low }}$ are only slightly changed by pressure and in the opposite sense, is difficult to understand in terms of the theories mentioned above, unless for example there is a rather unusual balance between several factors such as the pressure dependence of $E_{\mathrm{F}}$ and the electron-phonon coupling.

5.3 Our results above $T_{\text {HIGH }}$ lend some support to the suggestion [2] that electron-electron scattering is responsible for the dominant $T^{2}$ term in the resistivity. Under pressure the reduction in the $T^{2}$ term, together with the estimated increase in the transfer integral i. e. $E_{\mathrm{F}}$, are roughly consistent with the behaviour associated

(1) Denoyer, F. and Comes, R. (private communication). with electron-electron scattering [2]. Alternatively electron-phonon scattering with a pressure independent electron-phonon coupling constant will give rise to a conductivity proportional to $E_{\mathrm{F}}^{2}$ [1] which is also just consistent with the estimated increase in $E_{\mathrm{F}}$. Both of these conclusions are strongly dependent on the estimate of the compressibility of TTF-TCNQ.

\subsection{The nature of the intermediate region}

$$
T_{\text {LOW }}<T<T_{\text {HIGH }}
$$

is intriguing. Some of the features of the resistance measurements indicate a rather high anisotropy in this region (accounting for the large un-nested voltages in some samples, etc...), in general agreement with the results of reference [8]. However, the microscopic nature of this region cannot be established from the resistivity measurements alone. One possible interpretation of recent EPR measurements [15] is that in the metallic region the TTF and TCNQ chains contribute about equally to the conductivity, while in the semiconducting region (below $T_{\text {LOw }}$ ) the conductivity is due mostly to the TTF chains. In the intermediate region there is a continuous transition between the two extremes.

Based on this argument, we may venture to speculate that the transition at $T_{\text {HIGH }}$ affects mainly the TCNQ chains, and that electrical conductivity below $T_{\text {HIGH }}$ is mostly along TTF chains, isolated by the TCNQ chains, thus giving rise to a much larger anisotropy and dependence on defects (thus the vulnerability to thermal cycling), while at $T_{\text {Low }}$ the conductivity along the TTF chains in interrupted too, giving rise to a more or less normal semiconducting state. The possibility of a Fröhlich state [10] on the TCNQ chains in this temperature interval is also an interesting possibility.

Acknowledgments. - We are pleased to acknowledge Dr. E. M. Engler for preparing the samples and the technical assistance of G. Malfait and G. Delplanque. We are also grateful for numerous discussions with colleagues in our respective laboratories.

Notes added in proof. - (1) Some recent neutron work (Mook and Heeger private communication) enables the phonon velocity along the $b$ axis to be determined and shows that the estimate of the $b$-axis elastic constant we give in section 4 is wrong by about a factor of two. Furthermore, the approximation of neglecting the off-diagonal elastic constants is also dubious, so the estimate we give for the quantity $\alpha$ is at least a factor of two too high.

(2) Chu et al. [4] observed a small shoulder in the resistance of TTF-TCNQ at $45 \mathrm{~K}$ which disappeared under pressure. On the basis of this observation they suggested the possibility of separately conduc- 
ting TTF and TCNQ chains undergoing M-I transitions at slightly different temperatures which is similar to the interpretation given in our paper.
However, as can be seen from figure 1 (curves $A$ and $\mathrm{B}$ ) we also observed a shoulder at $45 \mathrm{~K}$ but it does not seem to be related to the lower transition.

\section{References}

[1] Jérome, D., Muller, W. and Weger, M., J. Physique Lett. 35 (1974) L 77.

[2] Etemad, S., Penney, T., Engler, E. M., Scott, B. A. and SeIden, P. E., Phys. Rev. Lett. 34 (1975) 741.

[3] Etemad, S., Penney, T. and Engler, E. M., Bull. Am. Phys. Soc. 496 March (1975);

Etemad, S. submitted to Phys. Rev.

The transition at $T_{\text {LOw }}$ was also reported recently by Berlinsky, A. J., Tiedje, T., Carolan, J. F., Weiler, L. and Friesen, W., Bull. Am. Phys. Soc. 465 March (1975).

[4] Chu, C. W., Harper, J. M. E., Geballe, T. H. and Greene, R. L., Phys. Rev. Lett. 31 (1973) 1491.

[5] Groff, R. P., Merrifield, R. E. and Suna, A. Phys. Rev. Lett. 33 (1974) 418.

[6] Ferraris, J. P., Cowan, D. O., Walatka, V. V. and Perlstein, J. E., J. Am. Chem. Soc. 95 (1973) 948.

[7] Craven, R. A., Salamon, M. B., De Pasquali, G., Herman, R. M., Stucky, G. and Schultz, A., Phys. Rev. Lett. 32 (1974) 769.

[8] Cohen, M. J., Coleman, L. B., Garito, A. F. and HeeGER, A. J., Phys. Rev. B 10 (1974) 1298.

[9] Schafer, D. E., Wudl, F., Thomas, G. A., Ferraris, J. P and Cowan, D. O., Solid Stat Commun. 14 (1974) 347.
[10] Allender, D., Bray, J. W. and Bardeen, J., Phys. Rev B 9 (1974) 119 ;

Weger, M., Horovitz, B. and Gutfreund, H., Phys. Rev. $B$. (Comments and Addenda) in press.

[11] Lee, P. A., Rice, T. M. and Anderson, P. W., Phys. Rev. Lett. 31 (1973) 462.

[12] Bernstein, U., Chaikin, P. M. and Pincus, P. Phys. Rev. Lett. 34 (1975) 271.

[13] Kuper, C. G., Proc. R. Soc. A 227 (1955) 214.

Gutfreund, H., Horowitz, B. and Weger, M., J. Physique colloq. 35 (1974) C 7-383 ; Phys. Rev. B 9 (1974) 1246 and Phys. Rev. B June (1975).

[14] Berlinsky, A. J., Carolan, J. F. and Weiler, L., Solid State Commun. 15 (1974) 795.

[15] Tomkiewicz, Y., Scott, B. A., TAO, L. J. and TitLE, R. S., Phys. Rev. Lett. 32 (1974) 1363.

[16] Barmatz, M., Testardi, L. R., Garito, A. F. and Heeger, A. J., Solid State Commun. 15 (1974), 1229.

[17] Blessing, R. M. and Coppens, P., Solid State Commun 15 (1974) 215 and Skelton, E. F., Bloch, A. N., FerraRIS, J. P. and Cowan, D. O., Phys. Lett. 47A (1974) 313.

[18] American Inst. of Physics Handbook, sect 4, 82, 3rd Ed., McGraw-Hill (1972). 